\title{
FRACTURA DE PENE CON LESIÓN ASOCIADA DE URETRA: PRESENTACIÓN DE UN CASO Y REVISIÓN DE LA LITERATURA
}

Patricio García Marchiñena, Leandro Capiel, Diego Juarez, Juan Liyo, Carlos Giudice, Guillermo Gueglio y Oscar Damia.

Servicio de Urología. Hospital Italiano de Buenos Aires. Buenos Aires. Argentina.

Resumen.- OBJETIVO: La fractura de pene es una lesión que responde habitualmente a un traumatismo cerrado que ocurre con el pene en erección. Cerca del 20-30\% de los casos en occidente, se asocian a lesión del cuerpo esponjoso. Presentamos el caso de un paciente atendido en nuestro servicio y realizamos una revisión de la literatura.

MÉTODOS: Se trata de un paciente de 42 años de edad, que luego de una relación sexual violenta, presentó una fractura de pene con lesión asociada de uretra. Se lo intervino quirúrgicamente, se constató la lesión de ambos

Patricio García Marchiñena

Hualfin, 833

i 1424 Buenos Aires. (Argentina).

patricio.garcia@hospitalitaliano.org.ar

宁

Trabajo recibido: 15 de enero 2008 
cuerpos cavernosos y la sección completa de de la uretra. Ambas lesiones fueron reparadas en el mismo acto quirúrgico.

RESULTADOS: El paciente evolucionó satisfactoriamente sin complicaciones y sin secuelas estéticas ni funcionales.

CONCLUSIONES: La fractura de pene con lesión asociada de uretra es una patología excepcional, cuya etiología más frecuente en occidente se debe a una relación sexual violenta. Una correcta anamnesis y un examen físico correcto son suficientes en la mayoría de los casos para hacer diagnóstico. El abordaje quirúrgico temprano y el cierre de la albuginea junto a la reparación de la uretra, permite alcanzar los mejores resultados.

Palabras clave: Fractura de pene. Sección de uretra. Tratamiento quirúrgico temprano.

Summary.- OBJECTIVE: Penile fracture is a rare lesion that occurs almost exclusively during erection. This lesion may be associated with rupture of the urethra in 20-30\% of the cases. We describe a case that has been treated at our institution and review the literature.

METHODS: A 42 years old patient suffered fracture of penis, with urethral section, during sexual intercourse. The patient underwent surgical exploration, the lesions of the corporal bodies and urethra were identified. Both lesions were repaired.

RESULTS: patient's recovery was satisfactory without complications or esthetical or functional sequelae.

CONCLUSIONS: Penile fracture with urethral section is an exceptional disease, the most frequent cause of which in occident is violent sexual activity. For diagnosis it is necessary in most cases a correct anamnesis and physical examination. Early surgical approach and closure of the albuginea's lesion and repair of the urethral lesion is the best way of treatment.

Keywords: Penile fracture. Section of the urethra. Early surgical treatment.

\section{INTRODUCCIÓN}

La fractura de pene, es una lesión habitualmente causada por un traumatismo cerrado que ocurre con el pene en erección. Se caracteriza por la ruptura de la túnica albugínea de uno o ambos cuerpos cavernosos, y cerca del 20-30 \% de los casos la lesión se extiende hasta el cuerpo esponjoso.

Su presentación clínica suele ser característica, y en la mayoría de los casos nos orienta al diagnóstico. El exa- men físico y los métodos diagnósticos pueden confirmar la lesión de la túnica albugínea, así como la presencia de lesiones asociadas.

Actualmente se defiende el tratamiento quirúrgico urgente, con drenaje del hematoma subcutáneo, rafia de la túnica albugínea y uretroplastía cuando exista una lesión uretral, lo que hace de radical importancia el reconocimiento por parte del urólogo de esta patología.

Presentamos el caso de un paciente que fue atendido en nuestro servicio y realizamos una revisión de la literatura.

\section{CASO CLÍNICO}

Paciente masculino de 42 años de edad sin antecedentes de importancia, consulta a la sala de emergencias de un centro de salud periférico por presentar dificultad para orinar, asociado a un gran hematoma que comprendía la región abdominal inferior.

Al interrogatorio, refirió haber sufrido un traumatismo a nivel genital durante una relación sexual horas antes de la consulta. Al impactar su pene contra el periné de su pareja, refiere haber oído un chasquido, luego del cual tuvo una detumescencia del pene inmediata.

Al examen físico, se evidenció un gran hematoma que comprometía la región infraumbilical, genital y perineal, se palpa a nivel de la cara latero ventral derecha del pene una solución de continuidad, y se constata la presencia de uretrorragia.

Se decide realizar una cistouretrografía retrógrada donde se evidencia fuga de contraste a nivel de uretra pén-

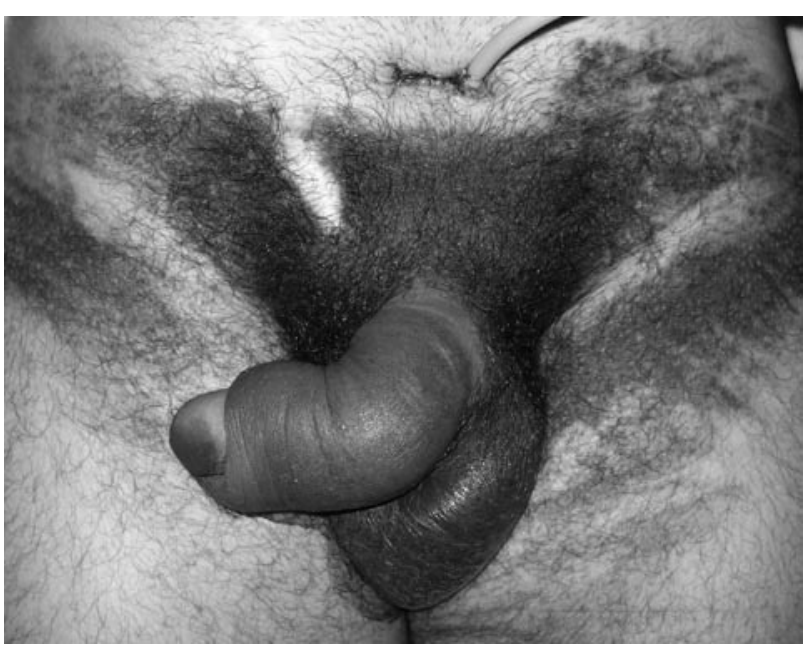

FIGURA 1. Hematoma en "alas de mariposa". 
dula. Se decide realizar una cistostomía percutánea y derivar al paciente a nuestro hospital.

Se recibe al paciente en la sala de emergencias, se constatan los hallazgos encontrados en el otro centro, se pide un laboratorio de rutina y una ecografía de pene mientras se aguardaba para llevar al paciente a quirófano.

En la ecografía se constató una disrupción de la túnica albugínea a nivel ventral de ambos cuerpos cavernosos y un gran hematoma asociado a edema de partes blandas a nivel latero ventral derecho.

Se realizó la exploración quirúrgica, denudando todo el pene a través de una incisión subcoronal, se evacuó un hematoma que se encontraba por encima de una solución de continuidad que abarcaba la cara ventral de ambos cuerpos cavernosos así como también la totalidad del cuerpo esponjosa. Se procedió a reparar la lesión de los cuerpos cavernosos con puntos simples de material reabsorbible. Luego se procedió a movilizar ambos cabos uretrales hasta lograr afrontarlos sin tensión, se espatularon los mismos en hora 6 y 12 respectivamente, ara así poder confeccionar una anastomosis termino terminal con material reabsorbible.

Luego de 48 horas de internación se le otorgó el alta hospitalaria con cistostomía abierta para el drenaje de orina y una sonda vesical cerrada para tutorizar la anastomosis. Bajo control radioscópico se retiró la sonda vesical a los 21 días de la cirugía, sin evidenciar fuga de contraste luego de realizar una cistouretrografía retrógrada de control.

A dos meses de la cirugía, el paciente se encuentra orinando normalmente, con erecciones conservadas y sin presencia de deformidades ni curvaturas a nivel peneano.

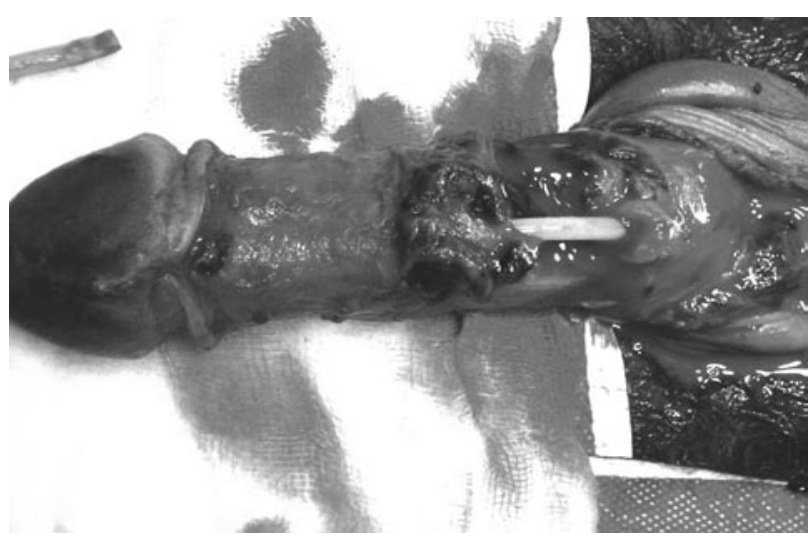

FIGURA 2. Disrupción uretral y rotura de túnica albugínea de ambos cuerpos cavernosos.

\section{DISCUSIÓN}

La fractura de pene es una lesión poco frecuente, que ocurre como resultado de un traumatismo cerrado, generalmente con el pene en erección, típicamente durante un acto sexual $(1,3,7,8)$.

La disminución del espesor que sufre la túnica albugínea durante la erección, sumado a un aumento brusco de la presión intracavernosa por la angulación forzada del pene, sería el mecanismo por el cual se produce la fractura $(1,4,5,8)$. La etiología más frecuente en occidente responde a traumatismos durante relaciones sexuales, siendo en países asiáticos más frecuente la fractura del pene secundaria a la manipulación del pene durante la masturbación, práctica conocida con el nombre de "taghaandan" $(1,5,8)$.

La asociación a ruptura de uretra varía de 0-3 \% en oriente (1), sin embargo en occidente los diferentes reportes muestran una asociación llamativamente mayor $(20-38 \%)(1,7,8,9)$, probablemente tenga que ver con la fuerza del traumatismo que lo genera.

El diagnóstico de la fractura de pene está basado en la anamnesis del paciente junto con el examen físico $(4,6,8)$. Clásicamente se presenta con dolor brusco, sensación de "chasquido", detumescencia peneana y desviación contra lateral del pene con posterior formación de hematoma, que dependerá de la indemnidad de la fascia de Back, si este se limita al pene o se extiende a todo el periné (en "alas de mariposa"). Si existe compromiso uretral podría referir dificultad para orinar, uretrorragia o hematuria $(4,5,7)$. Dentro de los diagnósticos diferenciales que deberían tenerse en cuenta está

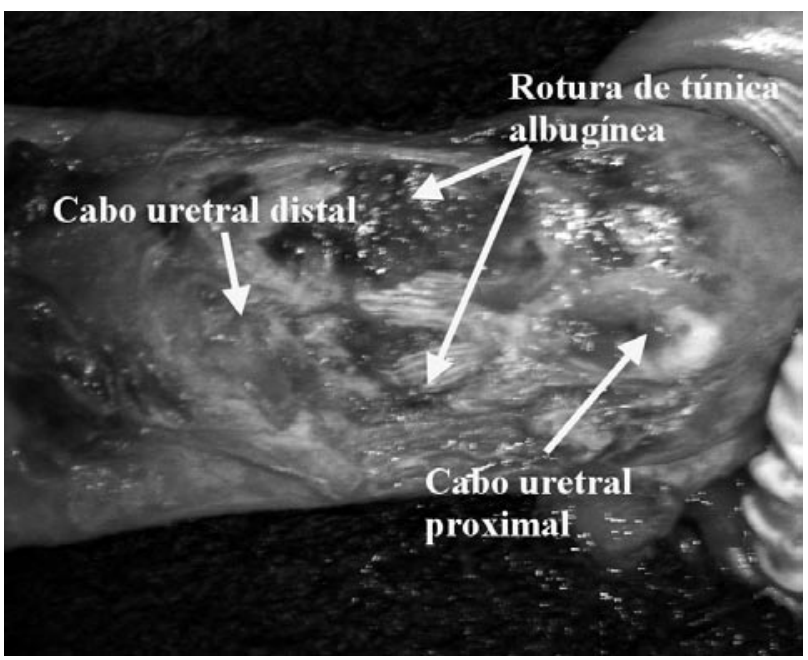

FIGURA 3. Magnificación de área de la lesión. 
la ruptura del ligamento suspensorio del pene y la ruptura de la vena dorsal profunda del pene, que pueden presentarse con similares síntomas $(1,8)$.

Se han propuesto varios estudios complementarios para el diagnóstico de esta patología, siendo la ecografía peneana, el de mayor aceptación. Tanto la cavernosografía por su invasividad, como la resonancia magnética por su costo y falta de disponibilidad en muchos hospitales, son métodos que no se utilizan rutinariamente. La uretrografía retrógrada está indicada, siempre que se sospeche una lesión asociada de uretra.

Debido a la alta tasa de complicaciones (29-40.7\%) que ha sido reportada con el tratamiento conservador (dolor, deformidad, impotencia, infección del hematoma, etc.) $(1,5,6)$, hoy en día se preconiza el tratamiento quirúrgico temprano, con el cual se alcanzarían los mejores resultados.

\section{CONCLUSIONES}

La fractura de pene con lesión asociada de uretra representa una entidad rara cuyo diagnóstico es eminentemente clínico. La reparación quirúrgica de ambas lesiones en forma temprana representa la mejor forma de tratamiento.

\section{BIBLIOGRAFÍA y LECTURAS RECOMENDADAS (*lectura de interés $y^{* *}$ lectura fundamental)}

*1. ZARGOSHI, J.: "Penile fracture in Kermanshah, Iran: Report of 172 cases". J. Urol., 164: 364, 2000.

**2. ZARGOSHI, J.: "Penile fracture in Kermanshah, Iran: The long-term results of surgical treatment". Br. J. Urol., 89: 890, 2002.

*3. MOUNTENER, M.; SUTER, S. y cols.: "Long-term experience with surgical and conservative treatment of penile fracture". J. Urol., 172: 576, 2004.

*4. MYDLO, J.; GERSHBEIN, A. y cols.: "Nonoperative treatment of patients with presumed penile fractura". J. Urol., 165: 424, 2001.

*5. EKE, N.: "Fracture of the penis (Review)". Br. J. Surg., 89: 555, 2002.

6. MYDLO, J.: "Surgeon experience with penile fracture". J. Urol., 166: 526, 2001.

*7. LLARENA IBARGUREN, R.; VILLAFRUELA, A. y cols.: "Fractura de pene con rotura asociada de uretra". Arch. Esp. Urol., 59: 732, 2006.

**8. PALACIOS, A.; MASSÓ, P. y cols.: "Fractura dos corpos cavernosos. A presentação de 9 casos". Act. Urol., 23: 41, 2006.

**9. HERRANZ, L.; ARELLANO, R. y cols.: "Fractura de pene". Arch. Esp. Urol., 56: 1079, 2003.

**10. MARTÍNEZ PÉREZ, E.; ARNAIZ ESTEBAN, F. y cols.: "Fractura de pene: Dos nuevos casos. Revisión de la literatura. Utilidad de la ecografía". Arch. Esp. Urol. 50: 1099, 1997. 